\title{
On Exact Series Solution of Strongly Coupled Mixed Parabolic Problems
}

\author{
Vicente Soler, ${ }^{1}$ Emilio Defez, ${ }^{2}$ M. V. Ferrer, ${ }^{3}$ and J. Camacho ${ }^{2}$ \\ ${ }^{1}$ Departamento de Matemàtica Aplicada, Universitat Politècnica de València, Camino de Vera S/N, 46022 Valencia, Spain \\ ${ }^{2}$ Instituto de Matemàtica Multidisciplinar, Universitat Politècnica de València, Camino de Vera S/N, 46022 Valencia, Spain \\ ${ }^{3}$ Departamento de Matemàtica e Informàtica, Universidad Jaume I de Castellón, Avenida de Vicent Sos Baynat S/N, \\ 12071 Castellón de la Plana, Spain
}

Correspondence should be addressed to Emilio Defez; edefez@imm.upv.es

Received 25 March 2013; Accepted 24 June 2013

Academic Editor: Juan Carlos Cortés López

Copyright (c) 2013 Vicente Soler et al. This is an open access article distributed under the Creative Commons Attribution License, which permits unrestricted use, distribution, and reproduction in any medium, provided the original work is properly cited.

This paper studies the construction of the exact solution for parabolic coupled systems of the type $u_{t}=A u_{x x}, A_{1} u(0, t)+B_{1} u_{x}(0, t)=$ $0, A_{2} u(l, t)+B_{2} u_{x}(l, t)=0,0<x<1, t>0$, and $u(x, 0)=f(x)$, where $A_{1}, A_{2}, B_{1}$, and $B_{2}$ are arbitrary matrices for which the block matrix $\left(\begin{array}{ll}A_{1} & B_{1} \\ A_{2} & B_{2}\end{array}\right)$ is nonsingular, and $A$ is a positive stable matrix.

\section{Introduction}

Coupled partial differential systems with coupled boundaryvalue conditions are frequent in quantum mechanical scattering problems [1-3], chemical physics [4-6], thermoelastoplastic modelling [7], coupled diffusion problems [8-10], and other fields. In this paper, we consider systems of the type

$$
\begin{gathered}
u_{t}(x, t)-A u_{x x}(x, t)=0, \quad 0<x<1, t>0, \\
A_{1} u(0, t)+B_{1} u_{x}(0, t)=0, \quad t>0, \\
A_{2} u(1, t)+B_{2} u_{x}(1, t)=0, \quad t>0, \\
u(x, 0)=f(x), \quad 0 \leq x \leq 1,
\end{gathered}
$$

where the unknown $u=\left(u_{1}, u_{2}, \ldots, u_{m}\right)^{T}$ and the initial condition $f=\left(f_{1}, f_{2}, \ldots, f_{m}\right)^{T}$ are $m$-dimensional vectors, $A_{i}, B_{i}, i=1,2$, are $m \times m$ complex matrices, elements of $\mathbb{C}^{m \times m}$, and $A$ is a matrix which satisfies the condition

$\operatorname{Re}(z)>0$ for all eigenvalues $z$ of $A$, and we say that $A$ is a positive stable matrix (where $\operatorname{Re}(z$ ) denotes the real part of $z \in \mathbb{C}$ ). We assume that the block matrix

$$
\left(\begin{array}{ll}
A_{1} & B_{1} \\
A_{2} & B_{2}
\end{array}\right) \text { is regular }
$$

and also that

the matrix pencil $A_{1}+\rho B_{1}$ is regular.

Condition (7) is well known from the literature of singular systems of differential equations, and it involves the existence of some $\rho_{0} \in \mathbb{C}$ such that matrix $A_{1}+\rho_{0} B_{1}$ is invertible [11].

Problem (1)-(4) with the less restrictive condition that (7) was solved in [12], but not with all of its blocks $A_{1}, A_{2}, B_{1}$, $B_{2}$, is singular (in particular $A_{1}=I$ ). Mixed problems of the previously mentioned type, but with the Dirichlet conditions $u(0, t)=0, u(1, t)=0$ instead of (2) and (3), have been treated in $[13,14]$.

Throughout this paper, and as usual, matrix $I$ denotes the identity matrix. The set of all the eigenvalues of a matrix $C$ in $\mathbb{C}^{m \times m}$ is denoted by $\sigma(C)$, and its 2 -norm $\|C\|$ is defined by $[15$, page 56$]$

$$
\|C\|=\sup _{x \neq 0} \frac{\|C x\|}{\|x\|},
$$


where for vector $y \in \mathbb{C}^{m}$, the Euclidean norm of $y$ is $\|y\|$. By $[15$, page 556$]$, it follows that

$$
\left\|e^{A t}\right\| \leq e^{t \alpha(A)} \sum_{k=0}^{m-1} \frac{\|\sqrt{m} A\|^{k} t^{k}}{k !}, \quad t \geq 0,
$$

where $\alpha(A)=\max \{\operatorname{Re}(w) ; w \in \sigma(A)\}$. We say that a subspace $E$ of $\mathbb{C}^{m}$ is invariant by the matrix $A \in \mathbb{C}^{m \times m}$, if $A(E) \subset$ $E$. If $B$ is a matrix in $\mathbb{C}^{n \times m}$, we denote by $B^{\dagger}$ its MoorePenrose pseudoinverse. A collection of examples, properties, and applications of this concept may be found in [11, 16], and $B^{\dagger}$ can be efficiently computed with the MATLAB and Mathematica computer algebra systems.

\section{Preliminaries and Notation}

In [17], eigenfunctions of problem (1)-(3) were constructed assuming other additional conditions besides (6) and (7). We recall in this section the notation and results needed. Let $\widetilde{A}_{1}$ and $\widetilde{B}_{1}$ be matrices defined by

$$
\widetilde{A}_{1}=\left(A_{1}+\rho_{0} B_{1}\right)^{-1} A_{1}, \quad \widetilde{B}_{1}=\left(A_{1}+\rho_{0} B_{1}\right)^{-1} B_{1},
$$

fulfilling the relation: $\widetilde{A}_{1}+\rho_{0} \widetilde{B}_{1}=I$. Under hypothesis (6), matrix $B_{2}-\left(A_{2}+\rho_{0} B_{2}\right) \widetilde{B}_{1}$ is regular; see [17, page 431], and let $\widetilde{A}_{2}$ and $\widetilde{B}_{2}$ be the matrices defined by

$$
\begin{aligned}
& \widetilde{A}_{2}=\left[B_{2}-\left(A_{2}+\rho_{0} B_{2}\right) \widetilde{B}_{1}\right]^{-1} A_{2}, \\
& \widetilde{B}_{2}=\left[B_{2}-\left(A_{2}+\rho_{0} B_{2}\right) \widetilde{B}_{1}\right]^{-1} B_{2},
\end{aligned}
$$

so that they satisfy the relationships

$$
\widetilde{B}_{2}-\left(\widetilde{A}_{2}+\rho_{0} \widetilde{B}_{2}\right) \widetilde{B}_{1}=I, \quad \widetilde{B}_{2} \widetilde{A}_{1}-\widetilde{A}_{2} \widetilde{B}_{1}=I .
$$

Assuming that the following condition

$$
\begin{aligned}
& \text { exists } b_{1} \in \sigma\left(\widetilde{B}_{1}\right)-\{0\}, \quad b_{2} \in \sigma\left(\widetilde{B}_{2}\right) \text {, } \\
& v \in \mathbb{C}^{m}-\{0\},
\end{aligned}
$$

such that $\left(\widetilde{B}_{1}-b_{1} I\right) v=\left(\widetilde{B}_{2}-b_{2} I\right) v=0$,

and that values $b_{1}, b_{2}$ of condition (13) satisfy

$b_{1} b_{2} \in \mathbb{R}, \quad$ where $b_{1} \in \mathbb{R}$ or $2 b_{1} b_{2}\left(\operatorname{Re}\left(b_{1}^{-1}\right)-\rho_{0}\right)=1$

$$
\text { if } b_{1} \notin \mathbb{R} \text {, }
$$

we can define the function

$$
\alpha\left(\rho_{0}, b_{1}, b_{2}, \lambda\right)=\frac{\left(1-b_{2}+\rho_{0} b_{1} b_{2}\right)\left(1-\rho_{0} b_{1}\right)}{b_{1}}-b_{1} b_{2} \lambda^{2},
$$

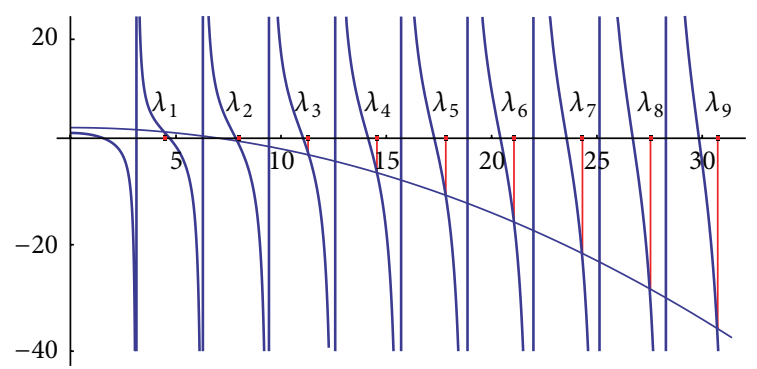

FIGURE 1: Graphical representation of $y=\lambda \cot (\lambda)$ and determination of the eigenvalues $\lambda_{n}$.

Note that under hypothesis (14) we have guaranteed the existence of the solutions for

$$
\lambda \cot (\lambda)=\frac{\left(1-b_{2}+\rho_{0} b_{1} b_{2}\right)\left(1-\rho_{0} b_{1}\right)}{b_{1}}-b_{1} b_{2} \lambda^{2} .
$$

Equation (16) has a unique solution $\lambda_{k}$ in each interval $(k \pi,(k+1) \pi)$ for $k \geq 1$, as seen in Figure 1. Also, it is straightforward to prove the following lemma.

Lemma 1. Under hypothesis (14), the roots $\lambda_{k}$ of (16) satisfy $\lim _{n \rightarrow \infty} \lambda_{n}=+\infty$. Also, if $b_{1} b_{2} \neq 0$, then

$$
\lim _{n \rightarrow \infty} \sin \left(\lambda_{n}\right)=0, \quad \lim _{n \rightarrow \infty}\left|\cos \left(\lambda_{n}\right)\right|=1 .
$$

Otherwise, if $b_{1} b_{2}=0$, then

$$
\lim _{n \rightarrow \infty}\left|\sin \left(\lambda_{n}\right)\right|=1, \quad \lim _{n \rightarrow \infty} \cos \left(\lambda_{n}\right)=0 .
$$

However, in all cases it is

$$
\lim _{n \rightarrow \infty}\left(\lambda_{n+1}-\lambda_{n}\right)=\pi
$$

Proof. Function $f(\lambda)=\lambda \cot (\lambda)$ has vertical asymptotes at the points $\lambda=k \pi, k \in \mathbb{N}$, and $f(\lambda)$ has zeros at the points $\lambda=(\pi / 2)+k \pi, k \in \mathbb{N}$. Thus, as we have stated, the real coefficient function $\left(\left(1-b_{2}+\rho_{0} b_{1} b_{2}\right)\left(1-\rho_{0} b_{1}\right) / b_{1}\right)-b_{1} b_{2} \lambda^{2}$ intersects the graph of the function $f(\lambda)$ in each interval $(k \pi,(k+1) \pi)$, where $\lambda_{k} \in(k \pi,(k+1) \pi)$ is the point of intersection. Thus, the sequence $\left\{\lambda_{k}\right\}_{k \geq 1}$ is monotonicaly increasing with $\lim _{k \rightarrow \infty} \lambda_{k}=\infty$. We have to consider two possibilities.

(i) $b_{1} b_{2}>0$. Function $\left(\left(1-b_{2}+\rho_{0} b_{1} b_{2}\right)\left(1-\rho_{0} b_{1}\right) / b_{1}\right)-$ $b_{1} b_{2} \lambda^{2}$ is therefore decreasing, and as seen in Figure 1 , for large enough $k$, then $\lambda_{k} \in((\pi / 2)+k \pi,(k+1) \pi)$.

(ii) $b_{1} b_{2}<0$. Function $\left(\left(1-b_{2}+\rho_{0} b_{1} b_{2}\right)\left(1-\rho_{0} b_{1}\right) / b_{1}\right)-$ $b_{1} b_{2} \lambda^{2}$ is therefore increasing, and as seen in Figure 1 , for large enough $k$, then $\lambda_{k} \in(k \pi,(\pi / 2)+k \pi)$.

Thus, observe that if $b_{1} b_{2} \neq 0$, then $(\pi / 2)<\lambda_{k+1}-\lambda_{k}<$ $(3 \pi / 2)$ for large sufficiently $k$. For $\lambda_{k}$, reemploying in (16), one gets

$$
\lambda_{k} \cot \left(\lambda_{k}\right)=\frac{\left(1-b_{2}+\rho_{0} b_{1} b_{2}\right)\left(1-\rho_{0} b_{1}\right)}{b_{1}}-b_{1} b_{2} \lambda_{k}^{2},
$$


dividing by $\lambda_{k}^{2}$ and taking limits where $k \rightarrow \infty$ :

$$
\lim _{k \rightarrow \infty} \frac{\cot \left(\lambda_{k}\right)}{\lambda_{k}}=-b_{1} b_{2} \neq 0 .
$$

This demonstrates that sequences $\left\{\lambda_{k}\right\}_{k \geq 1}$ and $\left\{\cot \left(\lambda_{k}\right)\right\}_{k \geq 1}$ are infinite equivalents and

$$
\lim _{k \rightarrow \infty} \cot \left(\lambda_{k}\right)=\infty
$$

where $\lim _{k \rightarrow \infty} \tan \left(\lambda_{k}\right)=0$. Moreover, as $\left\{\cos \left(\lambda_{k}\right)\right\}_{k \geq 1}$ is bounded, one gets that $\lim _{k \rightarrow \infty} \sin \left(\lambda_{k}\right)=0$ and $\lim _{k \rightarrow \infty}\left|\cos \left(\lambda_{k}\right)\right|=1$. Taking into account that

$$
\tan \left(\lambda_{k+1}-\lambda_{k}\right)=\frac{\tan \left(\lambda_{k+1}\right)-\tan \left(\lambda_{k}\right)}{1+\tan \left(\lambda_{k+1}\right) \tan \left(\lambda_{k}\right)},
$$

considering limits where $k \rightarrow \infty$, one gets $\lim _{k \rightarrow \infty} \tan \left(\lambda_{k+1}-\lambda_{k}\right)=0$, and with $(\pi / 2)<\lambda_{k+1}-\lambda_{k}<$ $(3 \pi / 2)$, then $\lim _{k \rightarrow \infty}\left(\lambda_{k+1}-\lambda_{k}\right)=\pi$.

If $b_{1} b_{2}=0$, then one obtains two possibilities.

(i) If $\left(\left(1-b_{2}+\rho_{0} b_{1} b_{2}\right)\left(1-\rho_{0} b_{1}\right) / b_{1}\right)>0$, as one can see in Figure 1, for large enough $k, \lambda_{k} \in(k \pi,(\pi / 2)+k \pi)$.

(ii) If $\left(\left(1-b_{2}+\rho_{0} b_{1} b_{2}\right)\left(1-\rho_{0} b_{1}\right) / b_{1}\right)<0$, as one can see in Figure 1, for large enough $k, \lambda_{k} \in((\pi / 2)+k \pi,(k+1) \pi)$.

Thus, observe that if $b_{1} b_{2}=0$, then also $(\pi / 2)<\lambda_{k+1}-$ $\lambda_{k}<(3 \pi / 2)$ for $k$ sufficiently large. For $\lambda_{k}$, reemploying in (16), one gets

$$
\lambda_{k} \cot \left(\lambda_{k}\right)=\frac{\left(1-b_{2}+\rho_{0} b_{1} b_{2}\right)\left(1-\rho_{0} b_{1}\right)}{b_{1}},
$$

dividing by $\lambda_{k}$ and taking limits where $k \rightarrow \infty$, one gets that $\lim _{k \rightarrow \infty} \cot \left(\lambda_{k}\right)=0$, and as the sequence $\left\{\sin \left(\lambda_{k}\right)\right\}_{k \geq 1}$ is bounded, one gets that $\lim _{k \rightarrow \infty} \cos \left(\lambda_{k}\right)=0$ and $\lim _{k \rightarrow \infty}\left|\sin \left(\lambda_{k}\right)\right|=1$. Moreover, one gets that

$$
\cot \left(\lambda_{k+1}-\lambda_{k}\right)=\frac{\cot \left(\lambda_{k+1}\right) \cot \left(\lambda_{k}\right)+1}{\cot \left(\lambda_{k}\right)-\cot \left(\lambda_{k+1}\right)},
$$

considering limits where $k \rightarrow \infty$, one gets

$$
\lim _{k \rightarrow \infty} \cot \left(\lambda_{k+1}-\lambda_{k}\right)=\infty,
$$

as the sequence $\left\{\cos \left(\lambda_{k+1}-\lambda_{k}\right)\right\}_{k \geq 1}$ is bounded, we have that $\lim _{k \rightarrow \infty} \sin \left(\lambda_{k+1}-\lambda_{k}\right)=0$, and with $(\pi / 2)<\lambda_{k+1}-\lambda_{k}<$ $(3 \pi / 2)$, one gets that $\lim _{k \rightarrow \infty}\left(\lambda_{k+1}-\lambda_{k}\right)=\pi$.

If $b_{1} b_{2}=0$ and $\left(\left(1-b_{2}+\rho_{0} b_{1} b_{2}\right)\left(1-\rho_{0} b_{1}\right) / b_{1}\right)=0$, (16) reduces to $\lambda \cot (\lambda)=0$, whose roots are $\lambda_{k}=(\pi / 2)+k \pi$, $k \in \mathbb{N}$, and trivially $\lambda_{k+1}-\lambda_{k}=\pi$. Then $\lim _{k \rightarrow \infty}\left(\lambda_{k+1}-\lambda_{k}\right)=$ $\pi$.

Under hypothesis $\alpha\left(\rho_{0}, b_{1}, b_{2}, \lambda_{0}\right)<1$ there is a root $\lambda_{0} \epsilon$ $(0, \pi)$, and we can define the set of eigenvalues of the problem (1)-(3) as

$$
\begin{aligned}
\mathscr{F}=\{ & \lambda_{k} \in(k \pi,(k+1) \pi) ; \\
& \left.\lambda_{k} \cot \left(\lambda_{k}\right)=\alpha\left(\rho_{0}, b_{1}, b_{2}, \lambda_{k}\right), k \geq 1\right\} \cup \mathscr{F}_{0},
\end{aligned}
$$

where

$$
\mathscr{F}_{0}= \begin{cases}\emptyset, & \text { if } \alpha\left(\rho_{0}, b_{1}, b_{2}, \lambda_{0}\right) \geq 1 \\ \lambda_{0} \in(0, \pi), & \text { if } \alpha\left(\rho_{0}, b_{1}, b_{2}, \lambda_{0}\right)<1 .\end{cases}
$$

Thus, by [17, page 433] a set of solutions of problem (1) is given by

$$
\begin{array}{r}
u\left(x, t, \lambda_{k}\right) \\
=e^{-\lambda_{k} A t}\left\{\sin \left(\lambda_{k} x\right) \widetilde{A}_{1}-\lambda_{k} \cos \left(\lambda_{k} x\right) \widetilde{B}_{1}\right\} C\left(\lambda_{k}\right), \\
\lambda_{k} \in \mathscr{F},
\end{array}
$$

where $C\left(\lambda_{k}\right)$ satisfies

$$
G\left(\rho_{0}, b_{1}, b_{2}, \lambda_{k}\right) C\left(\lambda_{k}\right)=0 .
$$

Observe that if $p$ is the degree of minimal polynomial of $A$, the matrix $G\left(\rho_{0}, b_{1}, b_{2}, \lambda_{k}\right)$ is defined by

$$
\begin{gathered}
G\left(\rho_{0}, b_{1}, b_{2}, \lambda_{k}\right) \\
=\left(\begin{array}{c}
\widetilde{B}_{1} A-A \widetilde{B}_{1} \\
\vdots \\
\widetilde{B}_{1} A^{p-1}-A^{p-1} \widetilde{B}_{1} \\
\left(\widetilde{A}_{2} \widetilde{A}_{1}+\lambda_{k}^{2} \widetilde{B}_{2} \widetilde{B}_{1}\right)+\alpha\left(\rho_{0}, b_{1}, b_{2}, \lambda_{k}\right) I \\
\left\{\left(\widetilde{A}_{2} \widetilde{A}_{1}+\lambda_{k}^{2} \widetilde{B}_{2} \widetilde{B}_{1}\right)+\alpha\left(\rho_{0}, b_{1}, b_{2}, \lambda_{k}\right) I\right\} A \\
\vdots \\
\left\{\left(\widetilde{A}_{2} \widetilde{A}_{1}+\lambda_{k}^{2} \widetilde{B}_{2} \widetilde{B}_{1}\right)+\alpha\left(\rho_{0}, b_{1}, b_{2}, \lambda_{k}\right) I\right\} A^{p-1}
\end{array}\right) .
\end{gathered}
$$

In order to ensure that $C\left(\lambda_{k}\right) \neq 0$ satisfies (30) we have

$$
\operatorname{rank} G\left(\rho_{0}, b_{1}, b_{2}, \lambda_{k}\right)<m,
$$

and under condition (32), the solution of (30) is given by

$C\left(\lambda_{k}\right)=\left(I-G\left(\rho_{0}, b_{1}, b_{2}, \lambda_{k}\right)^{\dagger} G\left(\rho_{0}, b_{1}, b_{2}, \lambda_{k}\right)\right) S, \quad S \in \mathbb{C}^{m}$.

The eigenfunctions associated to the problem (1) are then given by

$$
\begin{array}{r}
u\left(x, t, \lambda_{k}\right) \\
=e^{-\lambda_{k} A t}\left\{\sin \left(\lambda_{k} x\right) \widetilde{A}_{1}-\lambda_{k} \cos \left(\lambda_{k} x\right) \widetilde{B}_{1}\right\} C\left(\lambda_{k}\right), \\
\lambda_{k} \in \mathscr{F} .
\end{array}
$$

Also $\lambda=0$ is an eigenvalue of problem (1), if

$$
1 \in \sigma\left(-\widetilde{A}_{2} \widetilde{A}_{1}\right)
$$

Under hypothesis (35), if $G\left(\rho_{0}, 0\right)=\widetilde{A}_{2} \widetilde{A}_{1}+I$, then, if we denote by

$$
C(0)=\left(I-G\left(\rho_{0}, 0\right)^{\dagger} G\left(\rho_{0}, 0\right)\right) S, \quad S \in \mathbb{C}^{m},
$$


one gets that function

$$
u(x, 0)=\left(x \widetilde{A}_{1}-\widetilde{B}_{1}\right) C(0)
$$

is an eigenfunction of problem (1) associated to eigenvalue $\lambda=0$.

All these results are summarized in Theorem 2.1 of [17, page 434]. Our goal is to find the exact solution of the problem (1)-(4). We provide conditions for the function $f(x)$ and the matrix coefficients in order to ensure the existence of a series solution of the problem. The paper is organized as follows. In Section 3 a series solution for the problem is presented. In Section 4 we proceed with an algorithm and give an illustrative example.

\section{A Series Solution}

By the superposition principle, a possible candidate to the series solution of problem (1)-(4) is given by

$$
u(x, t)= \begin{cases}u(x, 0)+\sum_{\lambda_{n} \in \mathscr{F}} u\left(x, t, \lambda_{k}\right), & 0 \in \mathscr{F}, \\ \sum_{\lambda_{n} \in \mathscr{F}} u\left(x, t, \lambda_{k}\right), & 0 \notin \mathscr{F},\end{cases}
$$

where $u\left(x, t, \lambda_{k}\right)$ and $u(x, 0)$ are defined by (34) and (37), respectively, for suitable vectors $C\left(\lambda_{n}\right)$ and $C(0)$.

Assuming that series (38) and the corresponding derivatives $u_{x}(x, t), u_{x x}(x, t)$, and $u_{t}(x, t)$ are convergent (we will demonstrate this later), (38) will be a solution of (1)-(3). Now, we need to determine vectors $C(\lambda)$ and $C(0)$ so that (38) satisfies (4).

Note that, taking $v$ to satisfy (13), from (12) one gets

$$
\widetilde{A}_{2} v=\left(\frac{b_{2}-\rho_{0} b_{1} b_{2}}{b_{1}}\right) v, \quad \widetilde{A}_{1} v=\left(1-\rho_{0} b_{1}\right) v
$$

Under condition (39), we will consider the scalar SturmLiouville problem:

$$
\begin{gathered}
X^{\prime \prime}(x)+\lambda^{2} X(x)=0 \\
\left(1-\rho_{0} b_{1}\right) X(0)+b_{1} X^{\prime}(0)=0 \\
-\left(\frac{1-b_{2}+\rho_{0} b_{1} b_{2}}{b_{1}}\right) X(1)+b_{2} X^{\prime}(1)=0,
\end{gathered}
$$

which provides a family of eigenvalues $\mathscr{F}$ given in (27). Then, the associated eigenfunctions are

$$
\begin{gathered}
X_{\lambda_{n}}(x)=\left(1-\rho_{0} b_{1}\right) \sin \left(\lambda_{n} x\right)-b_{1} \lambda_{n} \cos \left(\lambda_{n} x\right), \quad \lambda_{n}>0, \\
X_{0}(x)=\left(1-\rho_{0} b_{1}\right) x-b_{1}, \quad \text { if } \lambda_{0}=0 .
\end{gathered}
$$

By the theorem of convergence of the Sturm-Liouville for functional series [18, chapter 11], with the initial condition $f(x)=\left(f_{1}(x), \ldots, f_{m}(x)\right)^{t}$ given in (4) satisfying the following properties:

$$
\begin{gathered}
f \in \mathscr{C}^{2}([0,1]), \\
\left(1-\rho_{0} b_{1}\right) f(0)+b_{1} f^{\prime}(0)=0, \\
-\left(\frac{1-b_{2}+\rho_{0} b_{1} b_{2}}{b_{1}}\right) f(1)+b_{2} f^{\prime}(1)=0,
\end{gathered}
$$

each component $f_{i}$ of $f$, for $1 \leq i \leq m$, has a series expansion which converges absolutely and uniformly on the interval $[0,1]$; namely,

$$
\begin{aligned}
f_{i}(x)= & \alpha\left(\left(1-\rho_{0} b_{1}\right) x-b_{1}\right) e_{0 i} \\
& +\sum_{\lambda_{n} \in \mathscr{F}}\left(\left(1-\rho_{0} b_{1}\right) \sin \left(\lambda_{n} x\right)-b_{1} \lambda_{n} \cos \left(\lambda_{n} x\right)\right) e_{\lambda_{n} i},
\end{aligned}
$$

where

$$
\begin{gathered}
\alpha=\left\{\begin{array}{l}
1 \quad \text { if } \frac{\left(1-b_{2}+\rho_{0} b_{1} b_{2}\right)\left(1-\rho_{0} b_{1}\right)}{b_{1}}=1 \\
0 \quad \text { if } \frac{\left(1-b_{2}+\rho_{0} b_{1} b_{2}\right)\left(1-\rho_{0} b_{1}\right)}{b_{1}} \neq 1,
\end{array}\right. \\
e_{0 i}=b_{1} \frac{\int_{0}^{1}\left(\left(1-\rho_{0} b_{1}\right) x-b_{1}\right) f_{i}(x) d x}{\int_{0}^{1}\left(\left(1-\rho_{0} b_{1}\right) x-b_{1}\right)^{2} d x} \quad \text { if } \lambda_{0}=0, \\
e_{\lambda_{n} i} \quad b_{1} \lambda_{n} \frac{\int_{0}^{1}\left(\left(1-\rho_{0} b_{1}\right) \sin \left(\lambda_{n} x\right)-b_{1} \lambda_{n} \cos \left(\lambda_{n} x\right)\right) f_{i}(x) d x}{\int_{0}^{1}\left(\left(1-\rho_{0} b_{1}\right) \sin \left(\lambda_{n} x\right)-b_{1} \lambda_{n} \cos \left(\lambda_{n} x\right)\right)^{2} d x} \\
\text { if } \lambda_{n}>0 .
\end{gathered}
$$

Thus,

$$
\begin{aligned}
f(x)= & \alpha\left(\left(1-\rho_{0} b_{1}\right) x-b_{1}\right) E(0) \\
& +\sum_{\lambda_{n} \in \mathscr{F}}\left(\left(1-\rho_{0} b_{1}\right) \sin \left(\lambda_{n} x\right)\right. \\
& \left.\quad-b_{1} \lambda_{n} \cos \left(\lambda_{n} x\right)\right) E\left(\lambda_{n}\right),
\end{aligned}
$$

where $E(0)=\left(\begin{array}{c}e_{01} \\ \vdots \\ e_{0 m}\end{array}\right)$ and $E\left(\lambda_{n}\right)=\left(\begin{array}{c}e_{\lambda_{n} 1} \\ \vdots \\ e_{\lambda_{n} m}\end{array}\right)$. On the other hand, from (38) and taking into account (34) and (37), one gets

$$
\begin{aligned}
f(x)= & u(x, 0)=\alpha\left(x \widetilde{A}_{1}-\widetilde{B}_{1}\right) C(0) \\
& +\sum_{\lambda_{n} \in \mathscr{F}}\left(\sin \left(\lambda_{n} x\right) \widetilde{A}_{1}-\lambda_{n} \cos \left(\lambda_{n} x\right) \widetilde{B}_{1}\right) C\left(\lambda_{n}\right) .
\end{aligned}
$$


We can equate the two expressions; if $C(0)$ and $C\left(\lambda_{n}\right)$, apart from conditions (33) and (36), satisfy $\{C(0), C(\lambda)\} \quad C$ $\operatorname{Ker}\left(\widetilde{B}_{1}-b_{1} I\right)$. Then, we have

$$
\begin{aligned}
& C\left(\lambda_{n}\right) \\
& =E\left(\lambda_{n}\right) \\
& =\frac{\int_{0}^{1}\left(\left(1-\rho_{0} b_{1}\right) \sin \left(\lambda_{n} x\right)-b_{1} \lambda_{n} \cos \left(\lambda_{n} x\right)\right) f(x) d x}{\int_{0}^{1}\left(\left(1-\rho_{0} b_{1}\right) \sin \left(\lambda_{n} x\right)-b_{1} \lambda_{n} \cos \left(\lambda_{n} x\right)\right)^{2} d x}, \\
& \begin{aligned}
C(0)=E(0) & \text { if } \lambda_{n}>0, \\
= & \frac{\int_{0}^{1}\left(\left(1-\rho_{0} b_{1}\right) x-b_{1}\right) f(x) d x}{\int_{0}^{1}\left(\left(1-\rho_{0} b_{1}\right) x-b_{1}\right)^{2} d x} \text { if } \lambda_{0}=0 .
\end{aligned}
\end{aligned}
$$

Note that $C(0)$ and $C(\lambda) \in \operatorname{Ker}\left(\widetilde{B}_{1}-b_{1} I\right)$, if

$$
f(x) \in \operatorname{Ker}\left(\widetilde{B}_{1}-b_{1} I\right) .
$$

Then $u(x, t)$ defined by

$$
\begin{aligned}
u(x, t)= & \alpha\left(\left(1-\rho_{0} b_{1}\right) x-b_{1}\right) C(0) \\
& +\sum_{\lambda_{n} \in \mathscr{F}} e^{-\lambda_{n}^{2} A t}\left(\left(1-\rho_{0} b_{1}\right) \sin \left(\lambda_{n} x\right)\right. \\
& \left.\quad-b_{1} \lambda_{n} \cos \left(\lambda_{n} x\right)\right) C\left(\lambda_{n}\right),
\end{aligned}
$$

where $\alpha$ and $C\left(\lambda_{n}\right)$ are defined by (44) and (47), satisfies the initial condition (4). Note that conditions (30)-(32) hold if

$$
G\left(\rho_{0}, b_{1}, b_{2}, \lambda_{k}\right) f(x)=0,
$$

and then

$$
\begin{gathered}
\left(\widetilde{B}_{1}-b_{1} I\right) A^{j} f(x)=0, \quad 0 \leq j<p, \\
\left(\widetilde{A}_{2} \widetilde{A}_{1}+\lambda_{n}^{2} \widetilde{B}_{2} \widetilde{B}_{1}+\alpha\left(\rho_{0}, b_{1}, b_{2}, \lambda\right) I\right) A^{j} f(x)=0, \\
0 \leq j<p .
\end{gathered}
$$

It is easy to check that conditions (48), (51) are equivalent to the condition

$$
A^{j} f(x) \in \operatorname{Ker}\left(\widetilde{B}_{1}-b_{1} I\right) \cap \operatorname{Ker}\left(\widetilde{B}_{2}-b_{2} I\right), \quad 0 \leq j<p .
$$

Condition (52) holds if

$$
\begin{gathered}
f(x) \in \operatorname{Ker}\left(\widetilde{B}_{1}-b_{1} I\right) \cap \operatorname{Ker}\left(\widetilde{B}_{2}-b_{2} I\right), \quad 0 \leq x \leq 1, \\
\operatorname{Ker}\left(\widetilde{B}_{1}-b_{1} I\right) \cap \operatorname{Ker}\left(\widetilde{B}_{2}-b_{2} I\right),
\end{gathered}
$$

is an invariant subspace with respect to matrix $A$.

Now we study the convergence of the solution given by (49) with $\alpha$ defined by (44) and $C\left(\lambda_{n}\right)$ by (47). Using Parseval's identity for scalar Sturm-Liouville problems [19], there exists a positive constant $M_{1}>0$ so that $\left\|C\left(\lambda_{n}\right)\right\| \leq M_{1}$. Taking formal derivatives in (49), one gets

$$
\begin{gathered}
\begin{aligned}
u_{t}(x, t)= & \sum_{\lambda_{n} \in \mathscr{F}}\left(-\lambda_{n}^{2}\right) e^{-\lambda_{n}^{2} A t} A\left(\sin \left(\lambda_{n} x\right)\left(1-\rho_{0} b_{1}\right)\right. \\
& \left.-\lambda_{n} \cos \left(\lambda_{n} x\right) b_{1}\right) C\left(\lambda_{n}\right), \\
u_{x}(x, t)= & \sum_{\lambda_{n} \in \mathscr{F}} \lambda_{n} e^{-\lambda_{n}^{2} A t}\left(\cos \left(\lambda_{n} x\right)\left(1-\rho_{0} b_{1}\right)\right. \\
& \left.+\lambda_{n} \sin \left(\lambda_{n} x\right) b_{1}\right) C\left(\lambda_{n}\right) \\
& +\alpha\left(1-\rho_{0} b_{1}\right) C(0), \\
u_{x x}(x, t)= & \sum_{\lambda_{n} \in \mathscr{F}} \lambda_{n}^{2} e^{-\lambda_{n}^{2} A t}\left(-\sin \left(\lambda_{n} x\right)\left(1-\rho_{0} b_{1}\right)\right. \\
& \left.+\lambda_{n} \cos \left(\lambda_{n} x\right) b_{1}\right) C\left(\lambda_{n}\right) .
\end{aligned}
\end{gathered}
$$

These series are all bounded in their respective norms:

$$
\begin{aligned}
& \|u(x, t)\| \\
& \leq \sum_{\lambda_{n} \in \mathscr{F}}\left[\left\|e^{-\lambda_{n}^{2} A t}\right\|\left|1-\rho_{0} b_{1}\right| M_{1}+\left\|\lambda_{n} e^{-\lambda_{n}^{2} A t}\right\|\left|b_{1}\right| M_{1}\right] \\
& \quad+\alpha\left(\left|1-\rho_{0} b_{1}\right| x+\left|b_{1}\right|\right)\|C(0)\|, \\
& \left\|u_{t}(x, t)\right\| \\
& \quad \leq \quad \sum_{\lambda_{n} \in \mathscr{F}}\left[\left\|\lambda_{n}^{2} e^{-\lambda_{n}^{2} A t} A\right\|\left|1-\rho_{0} b_{1}\right| M_{1}+\left\|\lambda_{n}^{2} e^{-\lambda_{n}^{2} A t}\right\|\left|b_{1}\right| M_{1}\right], \\
& \left\|u_{x}(x, t)\right\| \\
& \quad \leq \sum_{\lambda_{n} \in \mathscr{F}}\left[\left\|\lambda_{n}^{2} e^{-\lambda_{n}^{2} A t}\right\|\left|1-\rho_{0} b_{1}\right| M_{1}+\left\|\lambda_{n}^{2} e^{-\lambda_{n}^{2} A t}\right\|\left|b_{1}\right| M_{1}\right] \\
& \quad+\alpha\left|1-\rho_{0} b_{1}\right|\|C(0)\|, \\
& \left\|u_{x x}(x, t)\right\| \\
& \quad \leq \sum_{\lambda_{n} \in \mathscr{F}}\left[\left\|\lambda_{n}^{2} e^{-\lambda_{n}^{2} A t}\right\|\left|1-\rho_{0} b_{1}\right| M_{1}+\left\|\lambda_{n}^{3} e^{-\lambda_{n}^{2} A t}\right\|\left|b_{1}\right| M_{1}\right] .
\end{aligned}
$$

To check that the series is uniformly convergent in each domain $[0,1] \times[c, d]$, it is sufficient to verify that the series

$$
\sum_{\lambda_{n} \in \mathscr{F}} \lambda_{n}^{3} e^{-\lambda_{n}^{2} A t}
$$

is uniformly convergent in this domain. This is trivial because, using (9), one gets

$$
\left\|\lambda_{n}^{3} e^{-\lambda_{n}^{2} A t}\right\| \leq e^{-\lambda_{n}^{2} \alpha(A) t} \sum_{k=0}^{m-1} \frac{(\sqrt{m}\|A\| t)^{k} \lambda_{n}^{2 k+3}}{k !}
$$


and from the d'Alembert test series applied to each summand, taking into account (5) and the relation (19), $\lim _{n \rightarrow \infty}\left(\lambda_{n+1}-\right.$ $\left.\lambda_{n}\right)=\pi$, given in Lemma 1 , one gets for $3 \leq r \leq 2(m-1)+3$ that

$$
\begin{aligned}
\lim _{n \rightarrow \infty} e^{\left(\lambda_{n}^{2}-\lambda_{n+1}^{2}\right) \alpha(A) t}\left(\frac{\lambda_{n+1}}{\lambda_{n}}\right)^{r} \\
\leq \lim _{n \rightarrow \infty} e^{\left(\lambda_{n}^{2}-\lambda_{n+1}^{2}\right) \alpha(A) t}\left(\frac{n+2}{n}\right)^{r} \\
=e^{-\alpha(A) t \pi \lim _{n \rightarrow \infty}\left(\lambda_{n}+\lambda_{n+1}\right)}=0<1 .
\end{aligned}
$$

Thus, the series (56) is convergent.

Independence of the series solution (49) with respect to the chosen $\rho_{0} \in \mathbb{R}$ can be demonstrated using the same technique as given in [20].

We can summarize the results in the following theorem.

Theorem 2. Consider the homogeneous problem with homogeneous conditions (1)-(4) under hypotheses (5), (6), and (7) verifying conditions (13) and (14). Let $f(x)$ be a vectorial function satisfying (42). Let $\mathscr{F}$ be the set defined by (27), and let $G\left(\rho_{0}, b_{1}, b_{2}, \lambda_{k}\right)$ be the matrix defined by (31), taking as eigenvalues of problems $\lambda \in \mathscr{F}$ satisfying

$$
\operatorname{rank}\left(G\left(\rho_{0}, b_{1}, b_{2}, \lambda_{k}\right)\right)<m
$$

including the eigenvalue $\lambda=0$ if $1 \in \sigma\left(-\widetilde{A}_{2} \widetilde{A}_{1}\right)$, and taking as eigenfunctions $u\left(x, t, \lambda_{k}\right)$ defined by (34). Let $\alpha$ be given by (44) and vectors $C\left(\lambda_{n}\right)$ defined by (47). Then, $u(x, t)$, as defined in (49), is a series solution of problem (1)-(4).

\section{Algorithm and Example}

We can summarize the process to calculate the solution of the homogeneous problem with homogeneous conditions (1)-(4) in Algorithm 1.

Example 1. We will consider the homogeneous parabolic problem with homogeneous conditions (1)-(4), where the matrix $A \in \mathbb{C}^{4 \times 4}$ is chosen as

$$
A=\left(\begin{array}{cccc}
2 & 0 & 0 & -1 \\
1 & 2 & 1 & -2 \\
-1 & 0 & 2 & 1 \\
0 & 0 & 0 & 1
\end{array}\right)
$$

and the $4 \times 4$ matrices $A_{i}, B_{i}, i \in\{1,2\}$, are

$$
\begin{aligned}
A_{1}=\left(\begin{array}{llll}
0 & 0 & 0 & 0 \\
0 & 0 & 0 & 0 \\
0 & 0 & 1 & 0 \\
0 & 0 & 0 & 1
\end{array}\right), & A_{2}=\left(\begin{array}{llll}
0 & 1 & 0 & 0 \\
1 & 0 & 0 & 0 \\
0 & 0 & 0 & 1 \\
0 & 0 & 0 & 0
\end{array}\right), \\
B_{1}=\left(\begin{array}{llll}
1 & 0 & 0 & 0 \\
0 & 1 & 0 & 0 \\
0 & 0 & 0 & 0 \\
0 & 0 & 0 & 0
\end{array}\right), & B_{2}=\left(\begin{array}{llll}
1 & 0 & 0 & 0 \\
1 & 0 & 0 & 0 \\
0 & 0 & 1 & 0 \\
0 & 0 & 0 & 1
\end{array}\right) .
\end{aligned}
$$

Also, the vectorial valued function $f(x)$ will be defined as

$$
f(x)=\left(\begin{array}{c}
0 \\
x^{2}-1 \\
0 \\
0
\end{array}\right) \text {. }
$$

Observe that the method proposed in [12] cannot be applied to solve this problem.

We will follow Algorithm 1 step to step.

(1) Matrix $A$ satisfies the condition (5), because $\sigma(A)=$ $\{1,2\}$. That is, $A$ is positive stable.

(2) Each of the matrices $A_{i}, B_{i}, i \in\{1,2\}$, is singular, and the block matrix

$$
\left(\begin{array}{ll}
A_{1} & B_{1} \\
A_{2} & B_{2}
\end{array}\right)=\left(\begin{array}{llll|llll}
0 & 0 & 0 & 0 & 1 & 0 & 0 & 0 \\
0 & 0 & 0 & 0 & 0 & 1 & 0 & 0 \\
0 & 0 & 1 & 0 & 0 & 0 & 0 & 0 \\
0 & 0 & 0 & 1 & 0 & 0 & 0 & 0 \\
\hline 0 & 1 & 0 & 0 & 1 & 0 & 0 & 0 \\
1 & 0 & 0 & 0 & 1 & 0 & 0 & 0 \\
0 & 0 & 0 & 1 & 0 & 0 & 1 & 0 \\
0 & 0 & 0 & 0 & 0 & 0 & 0 & 1
\end{array}\right)
$$

is regular.

(3) Note that although $A_{1}$ is singular, taking $\rho_{0}=1 \in \mathbb{R}$, the matrix pencil

$$
A_{1}+\rho_{0} B_{1}=I_{4 \times 4}
$$

is regular. Therefore, we take $\rho_{0}=1$.

(4) By (10) we have

$$
\begin{gathered}
\widetilde{A_{1}}=\left(A_{1}+\rho_{0} B_{1}\right)^{-1} A_{1}=A_{1}, \\
\widetilde{B_{1}}=\left(A_{1}+\rho_{0} B_{1}\right)^{-1} B_{1}=B_{1} .
\end{gathered}
$$

(5) By (11) we have

$$
\begin{gathered}
\widetilde{A_{2}}=\left(B_{2}-\left(A_{2}+\rho_{0} B_{2}\right) \widetilde{B_{1}}\right)^{-1} A_{2}=\left(\begin{array}{cccc}
-1 & 0 & 0 & 0 \\
0 & -1 & 0 & 0 \\
0 & 0 & 0 & 1 \\
0 & 0 & 0 & 0
\end{array}\right), \\
\widetilde{B_{2}}=\left(B_{2}-\left(A_{2}+\rho_{0} B_{2}\right) \widetilde{B_{1}}\right)^{-1} B_{2}=\left(\begin{array}{cccc}
-1 & 0 & 0 & 0 \\
-1 & 0 & 0 & 0 \\
0 & 0 & 1 & 0 \\
0 & 0 & 0 & 1
\end{array}\right) .
\end{gathered}
$$

(6) We have $\sigma\left(\widetilde{B_{1}}\right)=\{0,1\}$ and $\sigma\left(\widetilde{B_{2}}\right)=\{0,1,-1\}$. Note that in this case the condition (13) holds because with $b_{1}=1$ and $b_{2}=0 \in \sigma\left(\widetilde{B}_{2}\right)$ there exists a common eigenvector $v \in \mathbb{C}^{4}, v=(0,1,0,0)^{t}$, and thus $\operatorname{Ker}\left(\widetilde{B}_{1}-\right.$ $I) \cap \operatorname{Ker}\left(\widetilde{B}_{2}\right) \neq(0,0,0,0)^{t}$. We are therefore in Case 1 of Algorithm 1. 
Input data: $A, A_{1}, A_{2}, B_{1}, B_{2} \in \mathbb{C}^{m \times m}, f(x) \in \mathbb{C}^{m}$.

Result: $u(x, t)$.

(1) Check that matrix $A$ satisfies (5).

(2) Check that matrices $A_{i}, B_{i} \in \mathbb{C}^{m \times m}, i \in\{1,2\}$ are singular, and check that the block matrix $\left(\begin{array}{ll}A_{1} & B_{1} \\ A_{2} & B_{2}\end{array}\right)$ is regular.

(3) Determine a number $\rho_{0} \in \mathbb{R}$ so that the matrix pencil $A_{1}+\rho_{0} B_{1}$ is regular.

(4) Determine matrices $\widetilde{A_{1}}$ and $\widetilde{B_{1}}$ defined by (10).

(5) Determine matrices $\widetilde{A_{2}}$ and $\widetilde{B_{2}}$ defined by (11).

(6) Consider the following cases:

(i) Case 1. Condition (13) holds, that is, matrices $\widetilde{B}_{1}$ and $\widetilde{B}_{2}$ have a common eigenvector $v \neq 0$ associated with eigenvalues $b_{1} \in \sigma\left(\widetilde{B}_{1}\right)-\{0\}$ and $b_{2} \in \sigma\left(\widetilde{B}_{2}\right)$. In this case continue with step (7).

(ii) Case 2. Condition (13) does not hold. In this case the algorithm stops because it is not possible to find the solution of (1)-(4) for the given data.

(7) Determine $b_{1} \in \sigma\left(\widetilde{B}_{1}\right), b_{1} \neq 0, b_{2} \in \sigma\left(\widetilde{B}_{2}\right)$ and vector $v \neq 0$ verifying

$v \in \operatorname{Ker}\left(\widetilde{B}_{1}-b_{1} I\right) \cap \operatorname{Ker}\left(\widetilde{B}_{2}-b_{2} I\right)$ such that:

(i) Conditions (53) hold, that is:

1.1: $\operatorname{Ker}\left(\widetilde{B}_{1}-b_{1} I\right) \cap \operatorname{Ker}\left(\widetilde{B}_{2}-b_{2} I\right)$ is an invariant subspace respect matrix $A$.

1.2: $f(x) \in \operatorname{Ker}\left(\widetilde{B}_{1}-b_{1} I\right) \cap \operatorname{Ker}\left(\widetilde{B}_{2}-b_{2} I\right), \forall x \in[0,1]$.

(ii) Conditions (14) hold, that is:

1.3: $\frac{\left(1-b_{2}+\rho_{0} b_{1} b_{2}\right)\left(1-\rho_{0} b_{1}\right)}{b_{1}} \in \mathbb{R}, b_{1} b_{2} \in \mathbb{R}$.

(iii) The vectorial function $f(x)$ satisfies (42), that is:

1.4: $f \in \mathscr{C}^{2}([0,1])$.

1.5: $\left(1-\rho_{0} b_{1}\right) f(0)+b_{1} f^{\prime}(0)=0$.

1.6: $-\left(\frac{1-b_{2}+\rho_{0} b_{1} b_{2}}{b_{1}}\right) f(1)+b_{2} f^{\prime}(1)=0$.

If these conditions are not satisfied, return to step (6) of Algorithm 1 discarding the values taken for $b_{1}$ and $b_{2}$.

(8) Determine the positive solutions of (16) and determine $\mathscr{F}$ defined by (27).

(9) Determine degree $p$ of minimal polynomial of matrix $A$.

(10) Building block matrix $G\left(\rho_{0}, b_{1}, b_{2}, \lambda_{k}\right)$ defined by (31).

(11) Determine $\lambda \in \mathscr{F}$ so that $\operatorname{rank} G\left(\rho_{0}, b_{1}, b_{2}, \lambda_{k}\right)<m$.

(12) Include the eigenvalue $\lambda=0$ if $1 \in \sigma\left(-\widetilde{A}_{2} \widetilde{A}_{1}\right)$.

(13) Determine $\alpha$ given by (44).

(14) Determine vectors $C\left(\lambda_{n}\right)$ defined by (47).

(15) Determine functions $u\left(x, t, \lambda_{n}\right)$ defined by (34).

(16) Determine the series solution $u(x, t)$ of problem (1)-(4) defined by (49).

Algorithm 1: Solution of the homogeneous problem with homogeneous conditions (1)-(4).

(7) We take the values $b_{1}=1$ and $b_{2}=0$ and will check the conditions given in step 7 of the algorithm.

(1.1) One gets that

$$
\operatorname{Ker}\left(\widetilde{B}_{1}-I\right) \cap \operatorname{Ker}\left(\widetilde{B}_{2}\right)=\left\langle\left(\begin{array}{l}
0 \\
1 \\
0 \\
0
\end{array}\right)\right\rangle .
$$

Let $x \in \operatorname{Ker}\left(\widetilde{B}_{1}-I\right) \cap \operatorname{Ker}\left(\widetilde{B}_{2}\right)$. Then $x=\left(\begin{array}{l}0 \\ \lambda \\ 0 \\ 0\end{array}\right)$, $\lambda \in \mathbb{C}$. In this case one gets

$$
A x=\left(\begin{array}{c}
0 \\
2 \lambda \\
0 \\
0
\end{array}\right) \in \operatorname{Ker}\left(\widetilde{B}_{1}-I\right) \cap \operatorname{Ker}\left(\widetilde{B}_{2}\right),
$$

and then the subspace $\operatorname{Ker}\left(\widetilde{B}_{1}-I\right) \cap \operatorname{Ker}\left(\widetilde{B}_{2}\right)$ is invariant by matrix $A$.

(1.2) It is trivial to check that

$$
f(x) \in \operatorname{Ker}\left(\widetilde{B}_{1}-I\right) \cap \operatorname{Ker}\left(\widetilde{B}_{2}\right), \quad \forall x \in[0,1] .
$$

(1.3) With these values $\rho_{0}, b_{1}$, and $b_{2}$, one gets that

$$
\frac{\left(1-b_{2}+\rho_{0} b_{1} b_{2}\right)\left(1-\rho_{0} b_{1}\right)}{b_{1}}=0 \in \mathbb{R} .
$$

With these values $b_{1}$ and $b_{2}$, one gets

$$
b_{1} b_{2}=0 \in \mathbb{R} \text {. }
$$

(1.4) It is trivial to check that $f(x) \in \mathscr{C}^{2}([0,1])$. 
(1.5) It is trivial to check that $\left(1-\rho_{0} b_{1}\right) f(0)+b_{1} f^{\prime}(0)=$ $(0,0,0,0)^{t}$.

(1.6) It is trivial to check that $-\left(\left(1-b_{2}+\right.\right.$ $\left.\left.\rho_{0} b_{1} b_{2}\right) / b_{1}\right) f(1)+b_{2} f^{\prime}(1)=(0,0,0,0)^{t}$.

(8) Equation (16) is of the form

$$
\lambda \cot (\lambda)=0
$$

We can solve (72) exactly, $\lambda_{k}=(\pi / 2)+k \pi$, with an additional solution $\left.\lambda_{0} \in\right] 0, \pi[$, because

$$
\frac{\left(1-b_{2}+\rho_{0} b_{1} b_{2}\right)\left(1-\rho_{0} b_{1}\right)}{b_{1}}=0<1,
$$

and then $\lambda_{0}=(\pi / 2)$. Thus, we have a numerable family of solutions of (72) which we denote by $\mathscr{F}$, given by.

$\mathscr{F}=\left\{\lambda_{k}=\frac{\pi}{2}+k \pi ; \lambda_{k} \in(k \pi,(k+1) \pi), k \geq 1\right\} \cup \mathscr{F}_{0}$,

$$
\mathscr{F}_{0}=\left\{\lambda_{0}=\frac{\pi}{2}\right\} .
$$

(9) The minimal polynomial of matrix $A$ is given by $p(x)=(x-2)^{3}(x-1)$. Then $p=4$.

(10) If $\lambda_{k}$ is a positive solution of (72), the matrix $G\left(\rho_{0}, b_{1}, b_{2}, \lambda_{k}\right)$ given by (31) takes the form

$$
G\left(1,1,0, \lambda_{k}\right)=\left(\begin{array}{cccc}
0 & 0 & 0 & -1 \\
0 & 0 & 1 & -2 \\
1 & 0 & 0 & 0 \\
0 & 0 & 0 & 0 \\
\hline 0 & 0 & 0 & -3 \\
0 & 0 & 4 & -6 \\
4 & 0 & 0 & 0 \\
0 & 0 & 0 & 0 \\
\hline 0 & 0 & 0 & -7 \\
0 & 0 & 12 & -13 \\
12 & 0 & 0 & 0 \\
0 & 0 & 0 & 0 \\
\hline-\lambda_{k}^{2} & 0 & 0 & 0 \\
-\lambda_{k}^{2} & 0 & 0 & 0 \\
0 & 0 & 0 & 1 \\
0 & 0 & 0 & 0 \\
\hline-2 \lambda_{k}^{2} & 0 & 0 & \lambda_{k}^{2} \\
-2 \lambda_{k}^{2} & 0 & 0 & \lambda_{k}^{2} \\
0 & 0 & 0 & 1 \\
0 & 0 & 0 & 0 \\
\hline-4 \lambda_{k}^{2} & 0 & 0 & 3 \lambda_{k}^{2} \\
-4 \lambda_{k}^{2} & 0 & 0 & 3 \lambda_{k}^{2} \\
0 & 0 & 0 & 1 \\
0 & 0 & 0 & 0 \\
\hline-8 \lambda_{k}^{2} & 0 & 0 & 7 \lambda_{k}^{2} \\
-8 \lambda_{k}^{2} & 0 & 0 & 7 \lambda_{k}^{2} \\
0 & 0 & 0 & 1 \\
0 & 0 & 0 & 0
\end{array}\right) .
$$

(11) Since the second column $G\left(1,1,0, \lambda_{k}\right)$ is zero, we have that $\operatorname{rank}\left(G\left(1,1,0, \lambda_{k}\right)\right)<4$. Thus, each one of the positive solutions given by (74) is an eigenvalue.

(12) It is trivial to check that $1 \notin \sigma\left(-\widetilde{A}_{2} \widetilde{A}_{1}\right)$, because

$$
-\widetilde{A}_{2} \widetilde{A}_{1}=\left(\begin{array}{cccc}
0 & 0 & 0 & 0 \\
0 & 0 & 0 & 0 \\
0 & 0 & 0 & -1 \\
0 & 0 & 0 & 0
\end{array}\right), \quad \sigma\left(-\widetilde{A}_{2} \widetilde{A}_{1}\right)=\{0\} .
$$

Then we do not include 0 as an eigenvalue.

(13) Taking into account that $\left(\left(1-b_{2}+\rho_{0} b_{1} b_{2}\right)(1-\right.$ $\left.\left.\rho_{0} b_{1}\right) / b_{1}\right)=0<1$, one gets $\alpha=0$.

(14) Vectors $C\left(\lambda_{n}\right)$ defined by (47) take the values

$$
C\left(\lambda_{n}\right)=\frac{64(-1)^{n}}{\pi^{4}(2 n+1)^{4}}\left(\begin{array}{l}
0 \\
1 \\
0 \\
0
\end{array}\right) \text {. }
$$

(15) Using the minimal theorem [21, page 571], one gets that

$e^{A u}=\left(\begin{array}{cccc}e^{2 u} & 0 & 0 & -e^{u}\left(e^{u}-1\right) \\ -\frac{1}{2} e^{2 u}(u-2) u & e^{2 u} & e^{2 u} u & \frac{1}{2} e^{u}\left(2+e^{u}(-2+(-2+u) u)\right) \\ -e^{2 u} u & 0 & e^{2 u} & e^{2 u} u \\ 0 & 0 & 0 & e^{u}\end{array}\right)$.

Next, by considering (78) with $u=-((\pi / 2)+n \pi)^{2} t$ and simplifying, we obtain the value of $e^{-((\pi / 2)+n \pi)^{2} A t}$. Taking into account that all eigenvalues $\lambda_{n}$ are positive, the associated eigenfunctions are

$$
\begin{aligned}
& u\left(x, t, \lambda_{n}\right) \\
& \quad=e^{-\lambda_{n}^{2} A t}\left(\left(1-\rho_{0} b_{1}\right) \sin \left(\lambda_{n} x\right)-b_{1} \lambda_{n} \cos \left(\lambda_{n} x\right)\right) C\left(\lambda_{n}\right) .
\end{aligned}
$$

(16) We replace the values of $C\left(\lambda_{n}\right)$ given by (77) in (79) and take into account the value of the matrix $e^{-((\pi / 2)+n \pi)^{2} A t}$. After simplification, we finally obtain the solution of (1)-(4) given by

$u(x, t)$

$$
\begin{aligned}
= & \left(\sum_{n \geq 0}-\frac{32(-1)^{n} e^{-(1 / 2)(\pi+2 n \pi)^{2} t} \cos ((1 / 2)(\pi+2 n \pi) x)}{\pi^{3}(2 n+1)^{3}}\right) \\
& \times\left(\begin{array}{l}
0 \\
1 \\
0 \\
0
\end{array}\right) .
\end{aligned}
$$

\section{Acknowledgments}

This research has been supported by the Universitat Politècnica de València Grant PAID-06-11-2020. The third listed author has been partially supported by the Universitat Jaume I, Grant P1.1B2012-05. 


\section{References}

[1] M. H. Alexander and D. E. Manolopoulos, "A stable linear reference potencial algorithm for solution of the quantum closecoupled equations in molecular scattering theory," Journal of Chemical Physics, vol. 86, pp. 2044-2050, 1987.

[2] V. S. Melezhik, I. V. Puzynin, T. P. Puzynina, and L. N. Somov, "Numerical solution of a system of integro-differential equations arising from the quantum mechanical three-body problem with Coulomb interaction," Journal of Computational Physics, vol. 54, no. 2, pp. 221-236, 1984.

[3] W. T. Reid, Ordinary Differential Equations, John Wiley \& Sons, New York, NY, USA, 1971.

[4] R. D. Levine, M. Shapiro, and B. Johnson, “Transition probabilities in molecular collisions: computational studies of rotational excitation," Journal of Chemical Physics, vol. 53, pp. 1755-1766, 1970.

[5] J. V. Lill, T. G. Schmalz, and J. C. Light, "Imbedded matrix Green's functions in atomic and molecular scattering theory," The Journal of Chemical Physics, vol. 78, no. 7, pp. 4456-4463, 1983.

[6] F. Mrugala and D. Secrest, "The generalized log-derivate method for inelastic and reactive collisions," Journal of Chemical Physics, vol. 78, pp. 5954-5961, 1983.

[7] T. Hueckel, M. Borsetto, and A. Peano, Modelling of Coupled Thermo-Elastoplastic Hydraulic Response of Clays Subjected to Nuclear Waste Heat, John Wiley \& Sons, New York, NY, USA, 1987.

[8] J. Crank, The Mathematics of Diffusion, Oxford University Press, 2nd edition, 1995.

[9] M. D. Mikhailov and M. N. Osizik, Unifield Analysis and Solutions of Heat and Mass Diffusion, John Wiley \& Sons, New York, NY, USA, 1984.

[10] I. Stakgold, Green's Functions and Boundary Value Problems, John Wiley \& Sons, New York, NY, USA, 1979.

[11] S. L. Campbell and C. D. Meyer Jr., Generalized Inverses of Linear Transformations, Pitman, London, UK, 1979.

[12] L. Jódar, E. Navarro, and J. A. Martin, "Exact and analyticnumerical solutions of strongly coupled mixed diffusion problems," Proceedings of the Edinburgh Mathematical Society II, vol. 43, no. 2, pp. 269-293, 2000.

[13] L. Jódar and E. Ponsoda, "Continuous numerical solutions and error bounds for time dependent systems of partial differential equations: mixed problems," Computers \& Mathematics with Applications, vol. 29, no. 8, pp. 63-71, 1995.

[14] E. Navarro, E. Ponsoda, and L. Jódar, "A matrix approach to the analytic-numerical solution of mixed partial differential systems," Computers \& Mathematics with Applications, vol. 30, no. 1, pp. 99-109, 1995.

[15] G. H. Golub and C. F. Van Loan, Matrix Computation, The Johns Hopkins University Press, Baltimore, Md, USA, 1989.

[16] C. R. Rao and S. K. Mitra, Generalized Inverse of Matrices and Its Applications, John Wiley \& Sons, New York, NY, USA, 1971.

[17] E. Navarro, L. Jódar, and M. V. Ferrer, "Constructing eigenfunctions of strongly coupled parabolic boundary value systems," Applied Mathematics Letters, vol. 15, no. 4, pp. 429-434, 2002.

[18] E. L. Ince, Ordinary Differential Equations, Dover, New York, NY, USA, 1962.

[19] E. A. Coddington and N. Levinson, Theory of Ordinary Differential Equations, McGraw-Hill, New York, NY, USA, 1967.
[20] V. Soler, E. Navarro, and M. V. Ferrer, "Invariant properties of eigenfunctions for multicondition boundary value problems," Applied Mathematics Letters, vol. 19, no. 12, pp. 1308-1312, 2006.

[21] N. Dunford and J. Schwartz, Linear Operators, Part I, Interscience, New York, NY, USA, 1977. 


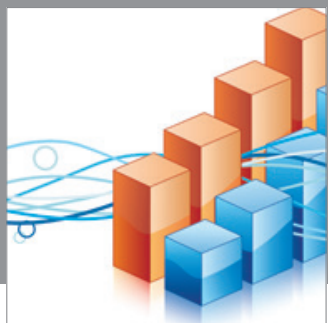

Advances in

Operations Research

mansans

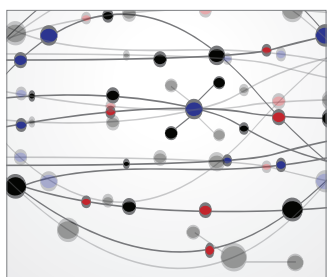

The Scientific World Journal
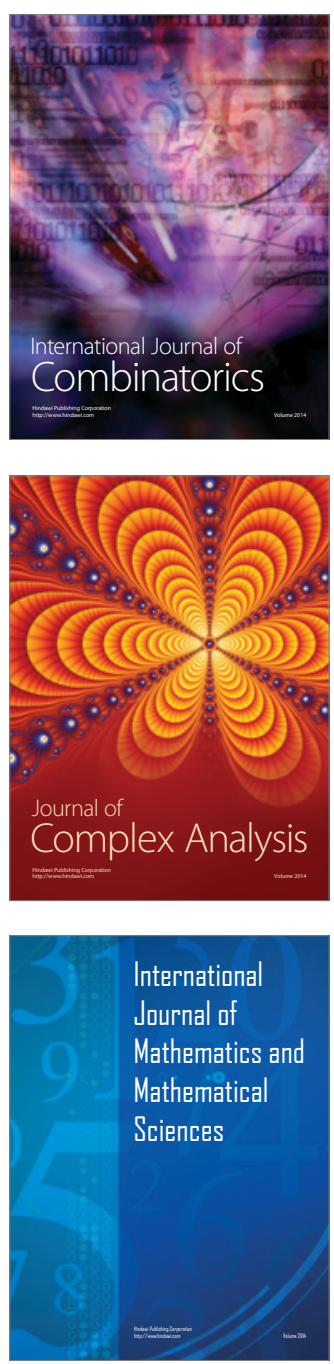
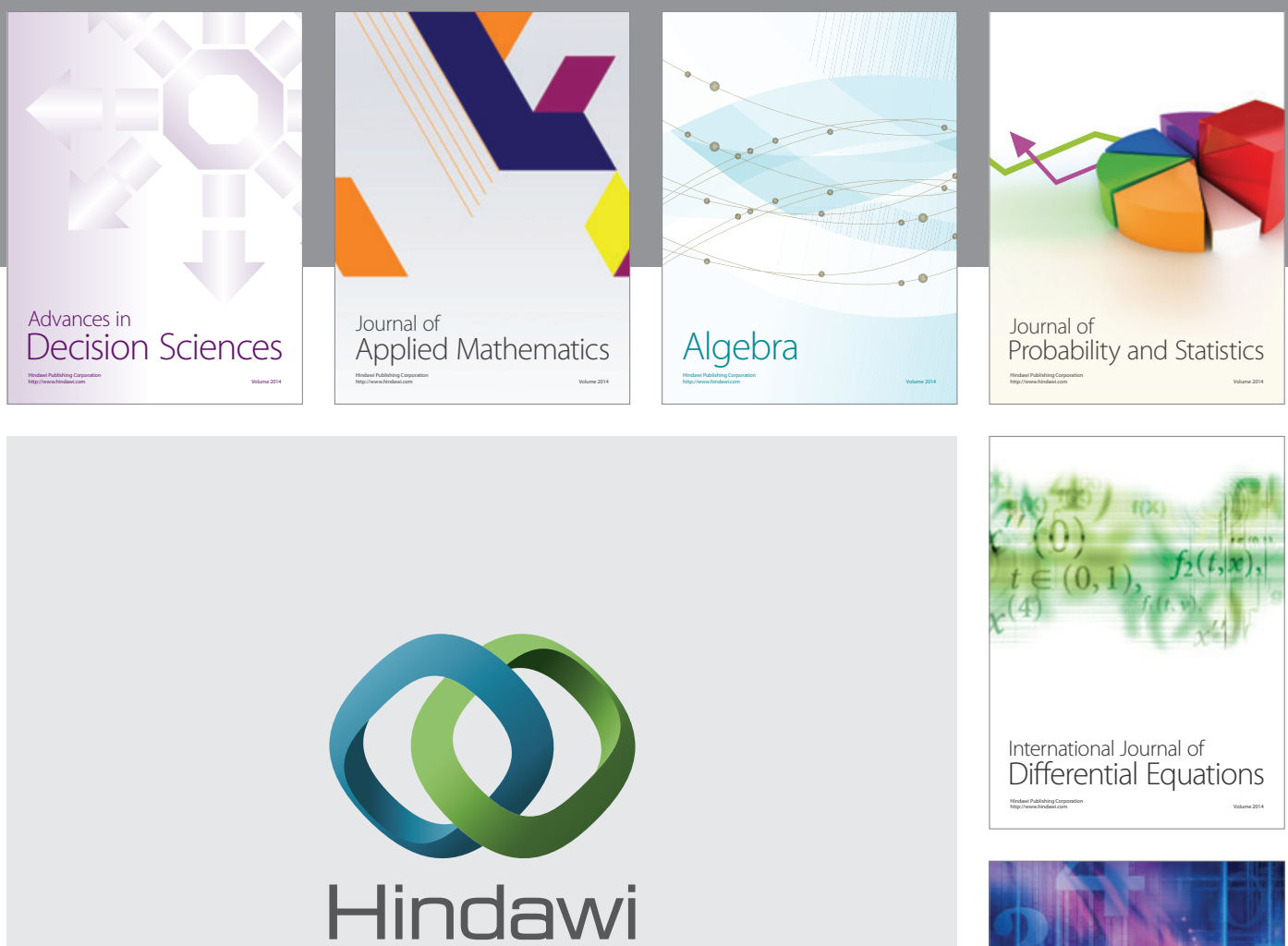

Submit your manuscripts at http://www.hindawi.com
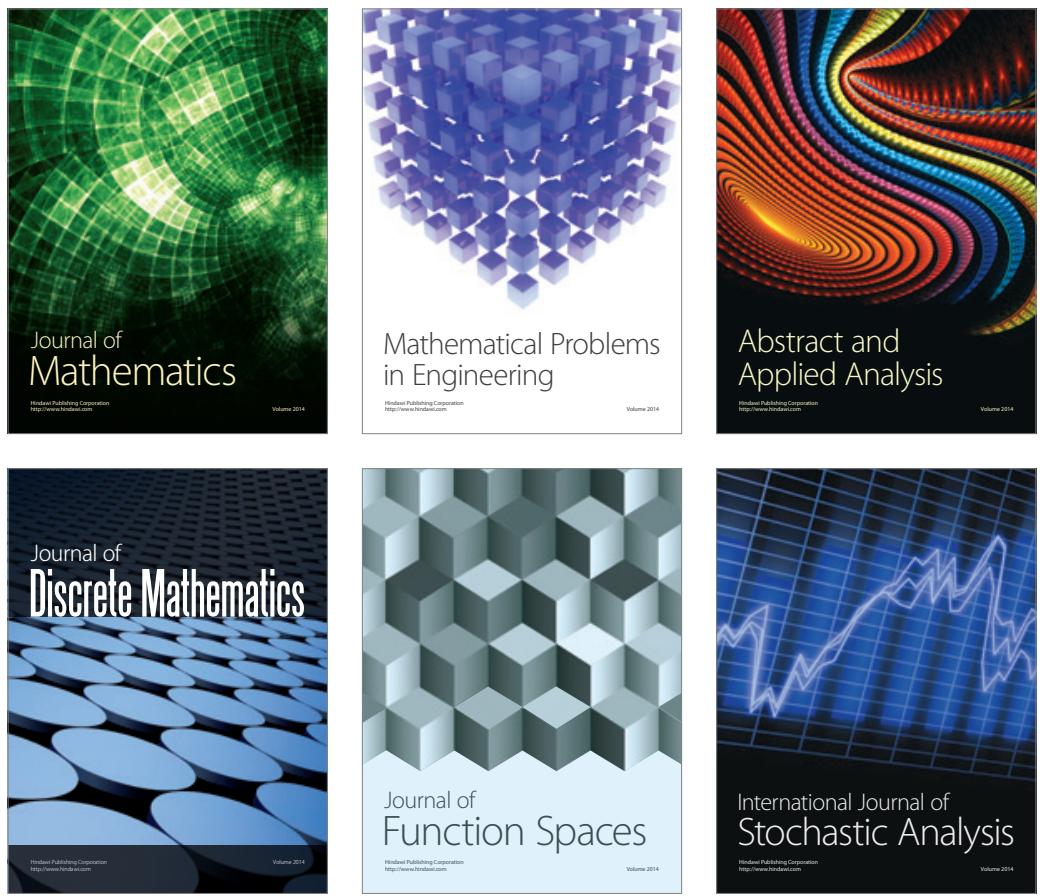

Journal of

Function Spaces

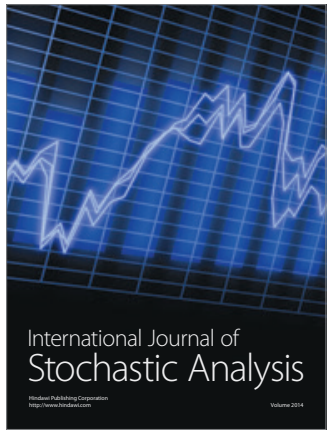

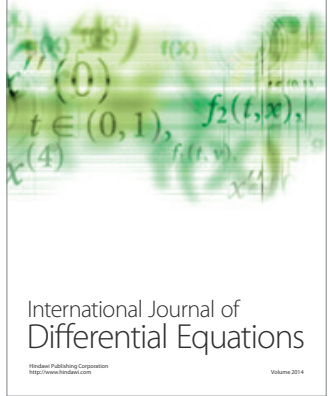
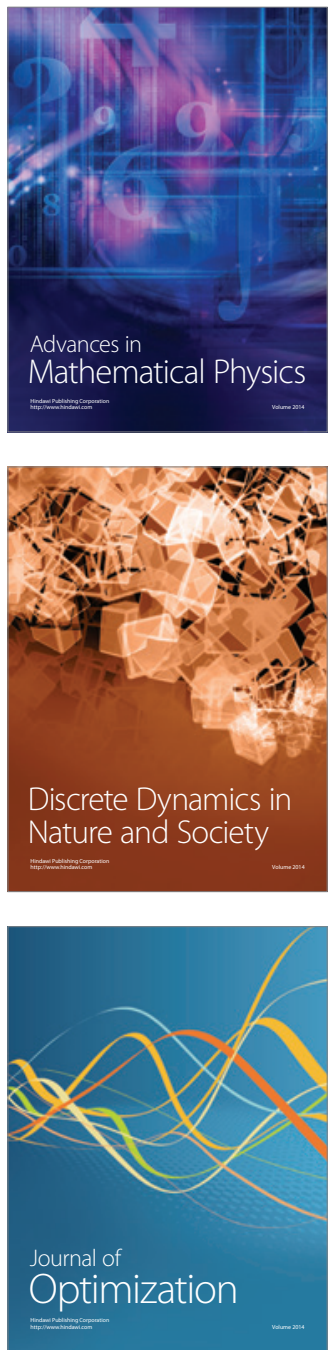